INDO GLOBAL JOURNAL OF

PHARMACEUTICAL SCIENCES

ISSN 2249- 1023

\title{
Bioinformatics Approaches for Genomics and Postgenomics Applications of Anticancer Patients
}

\author{
Avni Mehta, Yasha Hasija * \\ Department of Biotechnology, Delhi Technological University, Shahbad Daulatpur, Main Bawana Road, Delhi-110042
}

Address for Correspondence: Yasha Hasija, avnimehta28@gmail.com

\section{Keywords}

Bioinformatics; Anticancer; Plants;

Treatment; 'Omic' Technology.

\begin{abstract}
The combination of genomics, proteomics, bioinformstics, metabolomics and transcriptomics with robust ethnobotanical studies of traditional medicines may provide cues towards the development of effective treatments against cancer. 'Omic' technologies reveal differential effects of phytometabolites in anticancer plants such as Ranunculaceae on the phenotypically heterogeneous cancer cells. Derived from species like Ocimum, these metabolites have been found to have anticancer properties. Softwares such as STRING and IPA are used to analyse proteomic data to study molecular mechanisms of cancer remedies and to identify various signaling pathways. Bioinformatics tools are also used for the assembly and annotation of transcriptome of plants with anticancer properties. These include BLAST, FASTA, P-fam, Q-site finder, PyMOL, Modeller9v8, ChemSketch, etc. By using programs like Molinspiration miscreen engine that help in fast prediction of biological activity, a chemi-informatics approach may be adopted. Molecules of plant origin with anticancer traits are also tested by means of programs such as QikPro to analyse the pharmacokinetics potential and their ADME properties. The appearance of new bioinformatics methods, along with a growing range of 'omics' data, has opened vast perspectives in the study of pharmacological activity of plants. Protein docking simulations, clustering processes, machine-learning approaches and network pharmacology too have shown to be very effective. Genome-wide functional screening for promising pharmacological targets is also one of the most advanced and successful approaches in the post-genomic era. PathPred, CDRUG, PASS (Prediction for Active Spectra for Plants), Ingenuity Pathway Analysis and Cytoscape are some other sophisticated softwares that are frequently used. Thus, with the myriad of advances in 'omic' technologies, computational and in silico methodologies are cost-effective and efficient strategies to identify and utilize plant sources with anticancer properties. (C) 2016 iGlobal Research and Publishing Foundation. All rights reserved.
\end{abstract}

Conference Proceedings: International Conference on Advances in Plant and Microbial Biotechnology (PMB2017); JIIT, Noida: February 02-04, 2017

Indo Global Journal of Pharmaceutical Sciences( ISSN 22491023 ; CODEN- IGJPAI; NLM ID: 101610675) indexed and abstracted in EMBASE(Elsevier), SCIRUS(Elsevier),CABI, CAB Abstracts, Chemical Abstract Services(CAS), American Chemical Society(ACS), Index Copernicus, EBSCO, DOAJ, Google Scholar and many more. For further details, visit http://iglobaljournal.com 